\title{
Investigation of sensitivity for coagulation factor deficiency in APTT and PT: how to perform it?
}

DOI 10.1515/cclm-2015-0670

Received July 14, 2015; accepted October 5, 2015; previously published online November 20, 2015

Keywords: activated partial thromboplastin time (APTT); factor deficiency; factor sensitivity; prothrombin time (PT).

To the Editor,

The activated partial thromboplastin time (APTT) has been used for years for different purposes including screening for intrinsic factor deficiencies (FVIII, FIX, FXI and FXII) $[1,2]$. The APTT factor sensitivity depends on reagent composition comprising the type of contact activator as well as the origin and composition of phospholipids $[3,4]$. Current guidelines recommend a prolongation of the APTT when factor levels are below 30\% [5]. However, mild deficiencies above $30 \%$ can also imply a significant risk of bleeding $[6,7]$. Prothrombin time (PT) is primarily used to monitor oral vitamin K antagonists (VKA). Again, different reagents are available with different sensitivities [8]. Literature is very scarce but Testa et al. demonstrated a discrepant sensitivity to FV, FVII, and FX of two PT reagents [8]. Different sources of thromboplastins (rabbit, human, recombinant) may influence the FVII dosage [9]. Equally, a prolongation of the PT when factor levels drop from $30 \%$ to $45 \%$ is recommended [5].

By the use of dilution series of normal frozen plasma and freeze-dried or frozen factor deficient plasmas, the responsiveness of different APTT reagents has been tested [4]. Lawrie et al. suggested that lyophilized deficient plasmas may contain procoagulant material [10]. They concluded that determination of APTT factor sensitivity in

*Corresponding author: Katrien M.J. Devreese, Coagulation Laboratory, Department of Clinical Chemistry, Microbiology and Immunology, Ghent University Hospital, Ghent, Belgium, E-mail: katrien.devreese@uzgent.be

Els N. Dumoulin and Lisse Fiers: Coagulation Laboratory, Department of Clinical Chemistry, Microbiology and Immunology, Ghent University Hospital, Ghent, Belgium accordance with the CLSI guidelines can give inconsistent and misleading results. The use of well-defined patient samples was recommended [10].

Reagent composition and laboratory equipment are the most important variables for observed differences in sensitivity. Every laboratory should assess their own factor sensitivities in order to correctly interpret their routine coagulation tests. In this study, we aim to evaluate and compare the factor sensitivities in our daily practice by two appraoches: dilution experiments with normal pooled plasma (NPP) and in accordance with the CLSI guidelines by the use of patient deficient plasmas.

APTT (STA-PTTA, Diagnostica Stago, Asnières, France) and PT (Neoplastine CI Plus, Stago) were performed on the STA-R Evolution (Stago). One-stage-clotting assays were performed for intrinsic factors with STA-C.K. Prest (Stago) and for extrinsic factors with Neoplastine CI Plus (Stago), and by the use of lyophilized STA-Immunodeficient (STA-ID) (Stago) (FII, FVIII, FIX, FXI and FXII) and lyophilized STA-deficient (STA-D) (Stago) (FV, FVII, and FX) plasmas. In-house made NPP consisting of equal volumes of 80 healthy volunteers and stored at $-80^{\circ} \mathrm{C}$ was used for dilutions. Factor levels of NPP, STA-ID and STA-D plasmas were measured. Factor sensitivity of APTT (FVIII, FIX, FXI and FXI) and PT (FII, FV, FVII and FX) was assessed by two experiments. First, a spiking experiment was performed by mixing increasing volumes of NPP with factor deficient plasma. A series of 10 samples with factor activities of $10 \%$ up to $100 \%$ was obtained. Second, an analog series of \pm 22 patient samples was selected for every factor (two different patients per level). If certain factor activities lacked, different samples were mixed. A limited number of patients with FX deficiency and no patients with FII deficiency were available. APTT (s) or PT (s) and the studied factor (\%) were analyzed in duplicate whereas other intrinsic or extrinsic factors were analyzed in single. If factor levels were outside the reference range besides the studied factor, samples were excluded. A difference of $5 \%$ for duplicates was accepted [5]. The factor sensitivity is defined as the level of factor from where on a prolongation of APTT or PT was observed. Factor sensitivities were 
deduced graphically by evaluating the intersection of the upper limit of the reference range for APTT (38.1 s) or PT (16.5 s - in accordance with $70 \%)$ and the activity of the determined factor $[5,11]$. The study was approved by the Ethical Committee of the Ghent University Hospital and was in accordance with the Declaration of Helsinki.

All deficient plasmas showed factor levels below or equal to $2 \%$ for the factor of interest, as shown in Table 1. Other factors were within the normal range, except for STA-D FV plasma with $178 \%$ FVII and 5\% FVIII; the preparation of STA-D FV plasma by destabilization at room temperature, may explain that other labile factors such as FVIII will also be affected. FV and FVII levels in STA-ID FXII were borderline. All factor levels of the NPP were normal.

Results of the spiking experiments shown as dark grey squares are depicted in Figure $1 \mathrm{~A}$ and $\mathrm{B}$ for the intrinsic and extrinsic factors, respectively. The mean APTT or PT of the duplicate measurements was plotted on the $y$-axis, whereas the mean factor activity was plotted on the $\mathrm{x}$-axis. Evaluation showed an intrinsic factor sensitivity of $50 \%$ for FVIII, 52\% for FIX, 65\% for FXI and 46\% for FXII, and an extrinsic factor sensitivity of $36 \%$ for FII, 36\% for FV, 47\% for FVII and 41\% for FX. Factor sensitivity was also assessed by the use of patient samples, results are shown as light gray squares. Evaluation showed an intrinsic factor sensitivity of 55\% for FVIII, $69 \%$ for FIX, $81 \%$ for FXI and $78 \%$ for FXII, and an extrinsic factor sensitivity of 45\% for FV, 56\% for FVII and 53\% for FX.

In summary, results of our spiking experiment showed that factor sensitivities of the APTT were all above the upper limit of the proposed range of $30 \%-45 \%$ [5].
Consequently, mild (50\% FVIII, 52\% FIX, 46\% FXII) and for FXI very mild (65\%) deficiencies can be detected. The STA-PTTA reagent is the least sensitive for FXII (46\%) and the most for FXI (65\%). As FXII deficiencies are not associated with a bleeding tendency, and FXI levels do not correlate with bleeding risk, these findings are extremely useful. Patients with borderline FXI levels can present with a significant bleeding risk, therefore it is important to detect mild and very mild deficiencies [7]. In contrast to our finding, Bowyer et al. showed a FXI responsiveness for the STA-PTTA reagent of only $26 \mathrm{U} / \mathrm{dL}$ [4]. They used frozen immunodepleted FXI deficient plasma, commercial frozen NPP, and the CA7000 analyzer (Sysmex). These three variables lead to a contrasting result for FXI, again showing the importance of the whole setting. Besides, sensitivity ideally should be assessed by the use of patient deficient plasma [10]. Our experiments with patient plasmas showed markedly higher factor sensitivities for FIX, FXI and FXII compared to our spiking experiment. We cannot exclude that some of these discrepancies may be explained by factor deficiencies in the common coagulation pathway factors (FII, FV or FX). However, this seems very unlikely, since all samples originated from congenital single factor deficient patients. Though, results can be hampered by artifactial FV reduction due to pre-analytical sample quality.

Results for factor sensitivities of the PT were all in accordance with the CLSI guidelines [5]. The Neoplastine CI Plus reagent is the least sensitive for FII and FV (36\%) and the most for FVII (47\%). Very mild deficiencies will not be detected. Factor sensitivities established with patient samples are in accordance with the spiking experiment.

Table 1: Evaluation of the factor levels (\%) in STA-Immunodeficient (STA-ID), STA-deficient (STA-D) plasmas and in-house made normal pooled plasma (NPP).

\begin{tabular}{|c|c|c|c|c|c|c|c|c|}
\hline & FII & FV & FVII & FX & FVIII & FIX & FXI & FXII \\
\hline Reference range (\%) & $70-120$ & $70-120$ & $70-130$ & $70-120$ & $60-150$ & $60-150$ & $60-140$ & $60-150$ \\
\hline STA-ID FII & $<1$ & 78 & 85 & 79 & 70 & 118 & 89 & 90 \\
\hline STA-D FV & 80 & 2 & 178 & 71 & 5 & 67 & 71 & 94 \\
\hline STA-D FVII & 86 & 89 & 1 & 76 & 75 & 118 & 92 & 93 \\
\hline STA-D FX & 81 & 77 & 91 & $<1$ & 70 & 108 & 91 & 92 \\
\hline STA-ID FVIII & 82 & 81 & 73 & 75 & $<0.3$ & 94 & 78 & 73 \\
\hline STA-ID FIX & 82 & 85 & 82 & 79 & 83 & 0.9 & 78 & 76 \\
\hline STA-ID FXI & 93 & 87 & 86 & 89 & 75 & 93 & $<0.4$ & 71 \\
\hline STA-ID FXII & 74 & 67 & 66 & 76 & 60 & 101 & 74 & 1 \\
\hline NPP & 111 & 95 & 114 & 100 & 96 & 116 & 118 & 113 \\
\hline
\end{tabular}

Levels not in accordance with the reference ranges are shown in bold. NPP, normal pooled plasma; STA-D, STA-deficient; STA-ID, STA-immunodeficient. Shaded values correspond to the deficient factors of the commercial plasmas. 

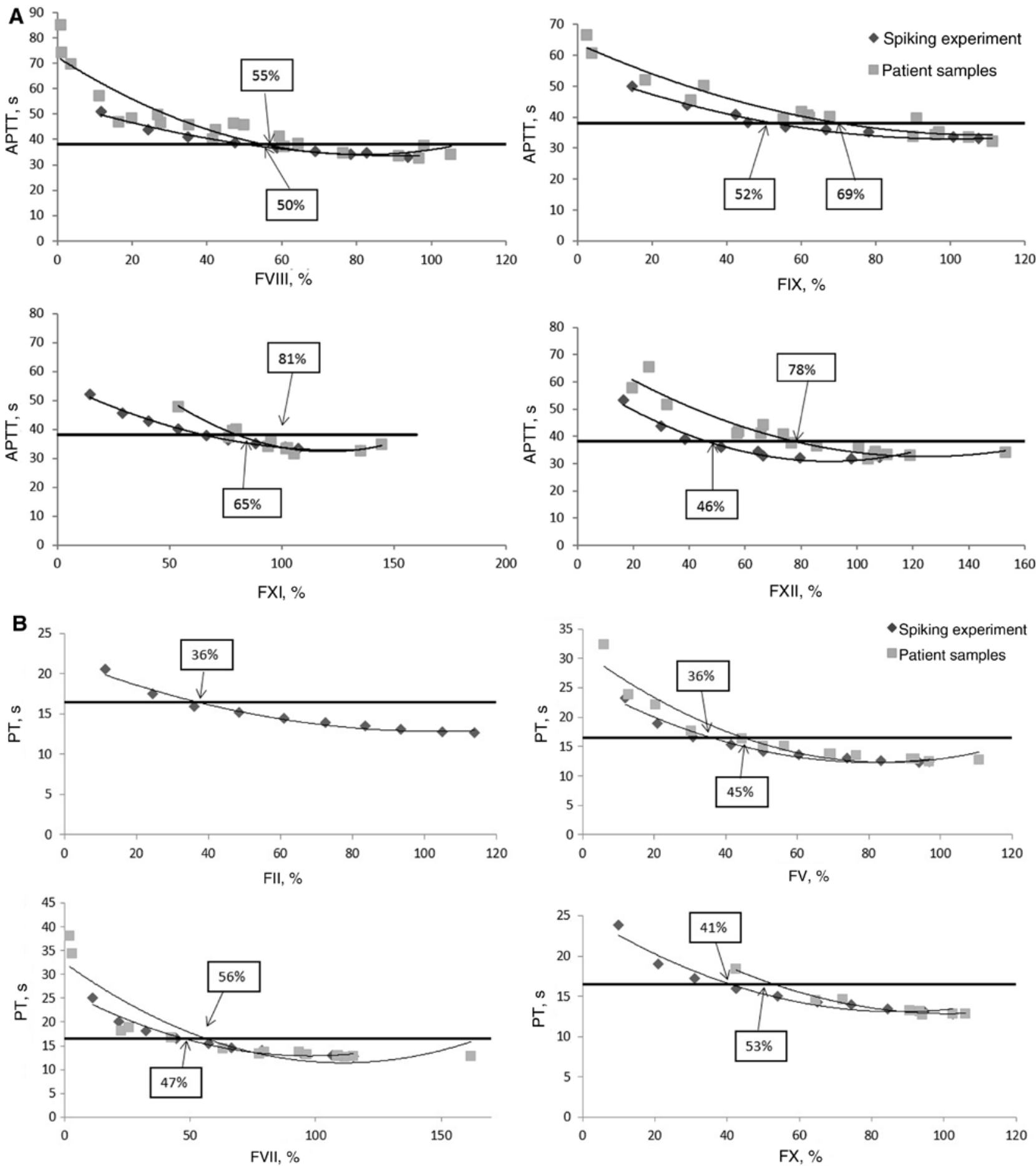

Figure 1: APTT and PT factor activity.

(A) Evaluation of APTT factor sensitivity: FVIII, FIX, FXI and FXII. Results of the spiking experiment are shown as dark gray diamonds ( $\diamond$ ), and results of the evaluation by patient samples are shown as light gray squares $(\square)$. APTT, activated partial thromboplastin time. (B) Evaluation of PT factor sensitivity: FII, FV, FVII and FX. Results of the spiking experiment are shown as dark gray diamonds ( $\diamond)$, and results of the evaluation by patient samples are shown as light gray squares ( ). PT, prothrombin time.

In conclusion, both the APTT (STA-PTTA) and the PT (Neoplastine CI Plus) tested in our setting show a very good factor sensitivity. Results obtained for PT sensitivity were comparable for both approaches. Discrepancy was observed only in the APTT. Collection, storage and availability of clinical samples are limitations in evaluating 
factor sensitivity with patient samples. Spiking experiments are easier to perform and more feasible for most laboratories. Of course, the results of this study should be carefully interpreted as factor sensitivity is depending on the whole laboratory setting.

Acknowledgments: We wish to thank Michael Luypaert for his technical support and all laboratory technicians of the Coagulation Laboratory for their practical help.

Author contributions: All the authors have accepted responsibility for the entire content of this submitted manuscript and approved submission.

Research funding: None declared.

Employment or leadership: None declared.

Honorarium: None declared.

Competing interests: The funding organization(s) played no role in the study design; in the collection, analysis, and interpretation of data; in the writing of the report; or in the decision to submit the report for publication.

\section{References}

1. Proctor RR, Rapaport SI. The partial thromboplastin time with kaolin. A simple screening test for first stage plasma clotting factor deficiencies. Am J Clin Pathol 1961;36:212-9.

2. van den Besselaar AM, Meeuwisse-Braun J, Bertina RM. Monitoring heparin therapy: relationships between the activated partial thromboplastin time and heparin assays based on ex-vivo heparin samples. Thromb Haemost 1990;63:16-23.

3. Pouplard C, Trossaert M, Le Querrec A, Delahousse B, Giraudeau B, Gruel Y. Influence of source of phospholipids for APTT-based factor IX assays and potential consequences for the diagnosis of mild haemophilia B. Haemophilia 2009;15:365-8.

4. Bowyer A, Kitchen S, Makris M. The responsiveness of different APTT reagents to mild factor VIII, IX and XI deficiencies. Int J Lab Hematol 2011;33:154-8.

5. (CLSI) Clinical and Laboratory Standards Institute. One-stage prothrombin (PT) test and activated partial thromboplastin time (APTT) test. H47-A2, 2008.

6. Plug I, Mauser-Bunschoten EP, Brocker-Vriends AH, van Amstel HK, van der Bom JG, van Diemen-Homan JE, et al. Bleeding in carriers of hemophilia. Blood 2006;108:52-6.

7. Bolton-Maggs PH, Patterson DA, Wensley RT, Tuddenham EG. Definition of the bleeding tendency in factor XI-deficient kindreds-a clinical and laboratory study. Thromb Haemost 1995;73:194-202.

8. Testa S, Morstabilini G, Fattorini A, Galli L, Denti N, D’Angelo A. Discrepant sensitivity of thromboplastin reagents to clotting factor levels explored by the prothrombin time in patients on stable oral anticoagulant treatment: impact on the international normalized ratio system. Haematologica 2002;87:1265-73.

9. Girolami A, Scarparo P, Bonamigo E, Treleani M, Lombardi AM. Homozygous FVII deficiencies with different reactivity towards tissue thromboplastins of different origin. Hematology 2012;17:350-4.

10. Lawrie AS, Kitchen S, Efthymiou M, Mackie IJ, Machin SJ. Determination of APTT factor sensitivity-the misguiding guideline. Int J Lab Hematol 2013;35:652-7.

11. Tripodi A, Lippi G, Plebani M. How to report results of prothrombin and activated partial thromboplastin times. Clin Chem Lab Med 2016;54:215-22. 